\title{
II est temps de prendre soin des soignants
}

\author{
Urs Brügger \\ Prof. Dr oec., membre de la rédaction
}

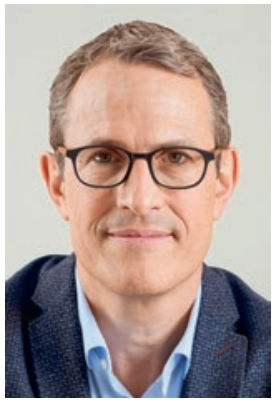

Dans les sondages, les médecins jouissent généralement d'un très haut degré de confiance au sein de la population par rapport à d'autres groupes professionnels. Mais les soignants font souvent encore mieux. Parmi le personnel infirmier, en revanche, la profession a de moins en moins la cote. Infirmières et infirmiers sont nombreux à se dire frustrés, surmenés, stressés, proches de l'épuisement voire déjà en burn-out. Il n'est pas rare d'en voir raccrocher et mettre prématurément un terme à leur carrière. "[Les] infirmières [sont] fatiguées et en colère», écrit l'Association suisse des infirmiers et infirmières (ASI) sur son site internet.

Sur le terrain, le corps infirmier a été en première ligne de la lutte contre la pandémie. Il a testé, trié, soigné, soutenu $24 \mathrm{~h}$ sur 24 . Souvent, c'est lui qui a accompagné les personnes isolées en fin de vie jusqu'à leur dernier souffle. Dans les hôpitaux, le facteur limitant n'était pas matériel: c'était le manque de personnel spécialisé, en particulier de personnel infirmier spécialisé.

Par ces propos «choc», l'ASI veut évidemment aussi attirer l'attention sur son initiative pour des soins infirmiers forts. Si certains points du texte suscitent le débat, tout le monde s'accorde pour dire qu'il faut faire quelque chose pour améliorer la situation des soins infirmiers et en garantir la qualité sur le long terme. En mars 2021, le Parlement a accepté le contre-projet indirect à l'initiative, dont il reprend des points importants. Il entend, d'une part, renforcer la formation de base et continue et, d'autre part, donner davantage d'autonomie au personnel infirmier pour qu'il puisse facturer certaines prestations sans prescription médicale.

En revanche, une autre revendication de l'initiative, l'amélioration des conditions de travail, n'a pas été reprise dans le contre-projet indirect, bien que fondamentale aux yeux de l'ASI pour qu'infirmiers et infirmières restent dans la profession. Former davantage de personnes ne suffit pas si celles-ci quittent le métier après quelques années. Yvonne Ribi, présidente de l'ASI, a dit à ce sujet: "C'est comme essayer de remplir une passoire avec de l'eau.»

L'initiative sur les soins infirmiers propose notamment de fixer un nombre maximum de patients soi-

gnés par soignant et d'inscrire dans la loi des règles pour une meilleure rémunération des soins. Personnellement, je ne pense pas que ce soit la bonne voie à suivre: c'est trop rigide et ne tient pas suffisamment compte des différentes réalités ni des évolutions futures. Il n'en reste pas moins urgent d'améliorer les conditions de travail.

Pour ce faire, je vois deux points essentiels : Premièrement, il s'agit d'engager infirmiers et infirmières conformément à leur formation et à leurs compétences. La profession a énormément évolué au cours des dernières années et compte toujours plus de titulaires d'un master ou d'un doctorat. Avec les APN (Advanced Practice Nurses, ou infirmières de pratique avancée), de nouveaux rôles voient le jour, aussi bien en milieu ambulatoire qu'hospitalier. Ces changements nécessitent urgemment des adaptations réglementaires et des mécanismes de financement afin de renforcer la profession et revaloriser le statut du personnel infirmier dans le système de santé.

Deuxièmement, le corps infirmier doit devenir un partenaire au même titre que les autres professions de la santé. C'est gaspiller les ressources que de ne pas impliquer correctement le groupe professionnel le plus proche des patients dans les décisions importantes liées à la gestion des hôpitaux ou la politique de santé. Groupe qui connaît parfaitement les processus et dispose de grandes compétences en management. Le personnel infirmier doit pouvoir faire entendre sa voix auprès des principales institutions et instances de la santé et obtenir plus d'influence et de pouvoir. Infirmiers et infirmières sont disposés à jouer un rôle de leader dans un contexte interprofessionnel.

Avec la pandémie de coronavirus, la population a encore davantage pris conscience de l'importance du personnel infirmier. Directions d'hôpitaux, médecins et politiques seraient donc bien inspirés d'accorder à ce groupe professionnel toute l'attention qu'il mérite. Cela permettrait de renforcer les soins comme notre système de santé et de lutter de manière efficace et durable contre la pénurie de personnel spécialisé dans les soins infirmiers. 